\title{
Comparative Phylogeography of Mesoamerican Highland Rodents: Concerted versus Independent Response to Past Climatic Fluctuations
}

\author{
Duke S. Rogers \\ duke_rogers@byu.edu \\ Jack Sullivan \\ Elizabeth Arellano
}

Follow this and additional works at: https://scholarsarchive.byu.edu/facpub

Part of the Biology Commons

\section{Original Publication Citation}

The American Naturalist 155.6(Jun 2): 755-768.

\section{BYU ScholarsArchive Citation}

Rogers, Duke S.; Sullivan, Jack; and Arellano, Elizabeth, "Comparative Phylogeography of Mesoamerican Highland Rodents: Concerted versus Independent Response to Past Climatic Fluctuations" (2001). Faculty Publications. 570.

https://scholarsarchive.byu.edu/facpub/570 


\section{Comparative Phylogeography of Mesoamerican Highland Rodents: Concerted versus Independent Response to Past Climatic Fluctuations}

\author{
Jack Sullivan, ${ }^{1, *}$ Elizabeth Arellano, ${ }^{2,3}$ and Duke S. Rogers \\ 1. Department of Biological Sciences, University of Idaho, \\ Moscow, Idaho 83844-3051; \\ 2. Department of Zoology and Monte L. Bean Life Science \\ Museum, Brigham Young University, Provo, Utah 84602; \\ 3. Centro de Educación Ambiente e Investigación Sierra de \\ Huautla, Universidad Autónoma del Morelos, Champila \\ Cuernavaca, Morelos 62210, México
}

Submitted June 2, 1999; Accepted January 15, 2000

ABSTRACT: The phylogeography of Sumichrast's harvest mouse (Reithrodontomys sumichrasti) was examined through maximumlikelihood and parsimony analyses of $1,130 \mathrm{bp}$ of mitochondrial Cytochrome $b$ sequence data from 43 individuals. The phylogeography of this Middle American highland forest-dwelling species was compared to that previously published for the codistributed Aztec deer mouse complex (Peromyscus aztecus/Peromyscus hylocetes complex) in order to test competing hypotheses of concerted versus independent responses of codistributed forms to past climatic fluctuations. Qualitatively, there were strong similarities in the phylogeographic patterns of the two groups, yet there were also areas of incongruence. Likelihood-ratio tests (Kishino-Hasegawa-Templeton and parametric bootstrap tests) indicated that this incongruence is significant and cannot be attributed simply to uncertainty in phylogenetic estimation, thereby falsifying the concerted-response hypothesis. Conversely, tree-reconciliation analysis of the area relationships inferred for each group separately indicated that there has been a significant history of covicariance between the two groups, falsifying the independent-response hypothesis. It appears that codistributed taxa in the geologically complex highlands of Mesoamerica share more common biogeographical history than can be accounted for by the independent-response hypothesis yet have not responded to past climatic fluctuations in the lock-step fashion predicted by the concerted-response hypothesis.

Keywords: comparative phylogeography, Middle American highlands, Peromyscus, parametric bootstrap, Reithrodontomys sumichrasti, maximum likelihood.

* To whom correspondence should be addressed; e-mail: jacks@uidaho.edu. Am. Nat. 2000. Vol. 155, pp. 755-768. (c) 2000 by The University of Chicago. 0003-0147/2000/15506-0004\$03.00. All rights reserved.
Fluidity of species' distributions during climatic fluctuations and associated glacial cycles in the Pleistocene has long been acknowledged. However, our understanding of the degree to which species that compose modern biotic communities have responded to these fluctuations en masse has changed dramatically. The classical paradigm has been that entire communities of organisms shifted in concert, both latitudinally and elevationally, in response to glacial cycles (e.g., Mengel 1964). Accumulating fossil data on mammal (Graham 1986; FAUNMAP 1996) and plant (e.g., Van Devender 1990a, 1990b) paleodistributions, however, suggest that community composition has been quite fluid. Indeed, it is now widely held that species ranges have changed independently in response to glacial cycles (Graham 1986; Bennett 1990; FAUNMAP 1996), and this view has become the dominant paradigm (e.g., Brown and Lomolino 1998).

If the independent-response hypothesis applies generally, there are at least two far-reaching evolutionary implications. First, little opportunity would have existed for long-term coevolution among community members. Because community membership would have been ephemeral, any coevolution that could currently be detected would have to have occurred quite rapidly. Second, a common practice in historical biogeography (e.g., Taberlèt et al. 1998) is to construct a general area cladogram, representing a single history of place, from congruence among area cladograms derived from phylogenetic analyses of multiple codistributed taxa; typically, such methods as Brooks's (1981) parsimony analyses are used. However, a prerequisite for such an approach is that codistributed taxa have responded to past geological and climatic events in a concerted fashion. Therefore, if generally true, the independent-response hypothesis would invalidate this approach to historical biogeography because any congruence among single-taxon area cladograms would be solely the result of chance rather than reflecting a single history of 


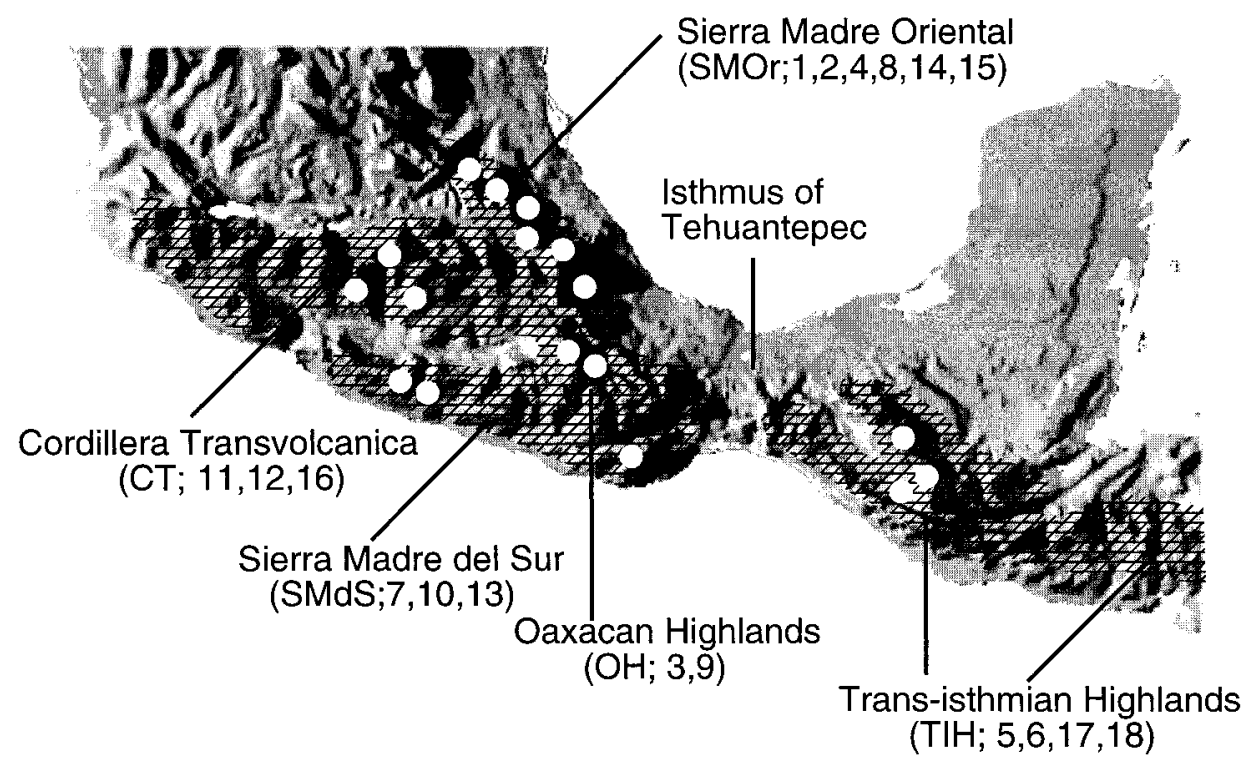

Figure 1: Distribution of Reithrodontomys sumichrasti and collection localities for samples examined here. Note that the Costa Rican portion of the distribution and the collection locality for the sample from Costa Rica are not on this map.

place. Using Brooks's parsimony analysis in such a case would be analogous to conducting phylogenetic analysis on random data; a single phylogenetic tree would possibly be obtained, but it would be meaningless.

Comparative phylogeography (Avise 1992, 1998) provides a means by which to distinguish these two paradigms (Riddle 1996, 1998); such studies assess the degree to which the members of particular biotic assemblages have responded to climatic and geographic events in concert (Zink 1996; Avise 1998). Furthermore, detection of congruent phylogeographies provides predictions about patterns of genetic variation for codistributed taxa that have yet to be sampled (Avise 1998) and, thereby, allows for the circumscription of regional conservation units from a multitaxon perspective (i.e., Avise 1992). Conversely, any incongruence detected among phylogeographies of codistributed taxa may result from ecological differences among the species under consideration (i.e., Avise 1998). Thus, comparative phylogeography provides a critical link that allows integration of historical and ecological biogeography (Riddle 1996).

The Middle American highlands represent an ideal model system for testing concerted- versus independentresponse hypotheses of codistributed species to past climatic and geologic events. These highlands include several distinct mountain ranges in central and southern Mexico and upper Middle America, and the region is characterized by very high rates of endemism (e.g., Fa and Morales 1993; Flores-Villela and Gerez 1994). The mountain ranges in- clude the Sierra Madre Oriental (SMOr), which extends north-south in eastern Mexico; the Cordillera Transvolcanica (CT), which runs east to west through central Mexico; the Sierra Madre del Sur (SMdS) along the southwestern coast of Mexico; the complex highlands in central Oaxaca $(\mathrm{OH})$; and the mountains of Chiapas and northwestern Middle America (TIH; fig. 1). Cloud and pine/ oak forests occur at moderate to high elevations in each of these ranges, and these forests are currently rendered disjunct by intervening wet or dry lowlands. Although paleoecological data for this region are too sparse to evaluate whether these forests were formerly more extensive, their present disjunct occurrence may be a result of fragmentation (Toledo 1982; McDonald 1993).

Two rodent species complexes, the Aztec deer mouse complex (Peromyscus aztecus/Peromyscus hylocetes complex) and Sumichrast's harvest mouse (Reithrodontomys sumichrasti) are broadly codistributed in several disjunct sets of populations in this region. For each complex, the fragmented ranges have been thought to represent remnants of once continuous montane species (i.e., Carleton 1979). Sullivan et al. (1997) examined the phylogeography of the P. aztecus/P. hylocetes complex and postulated a set of area relationships for the highlands of Mesoamerica (fig. 2). The classical paradigm, represented by the concertedresponse hypothesis, predicts that area relationships inferred from the codistributed $R$. sumichrasti will be congruent to those inferred from the $P$. aztecus/ $P$. hylocetes complex. Conversely, the current paradigm, represented 


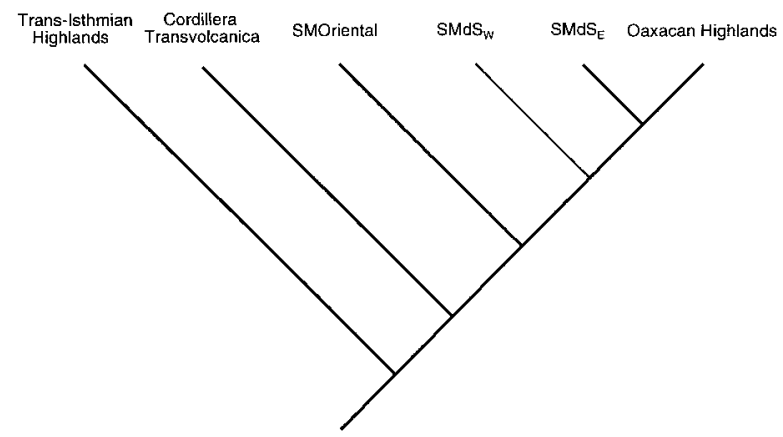

Figure 2: Hypothesis of area relationships for the Middle American highlands inferred by Sullivan et al. (1997) from examination of the phylogeography of the Peromyscus aztecus/Peromyscus hylocetes complex. The hypotheses of Sullivan et al. (1997) were augmented here by the addition of the sample of $P$. aztecus evides from Omiltemi, Guerrero, Mexico $\left(\mathrm{SMdS}_{\mathrm{E}}\right)$.

by the independent-response hypothesis, predicts no more congruence than would be expected by chance. Here, we use sequence data from the mitochondrial Cytochrome $b$ gene (cyt b) to test the predictions of both the concertedresponse hypothesis and the independent-response hypothesis for these two highland rodent complexes.

\section{Methods}

Samples

Forty-three individuals of Sumichrast's harvest mice were examined from among the 18 localities listed in table 1. At least one sample is included from each of the three allopatric segments of the distribution; however, populations in the mountains of southcentral Mexico are more thoroughly sampled than any from southeast of the Isthmus of Tehuantepec (fig. 1). In addition, Reithrodontomys megalotis and Reithrodontomys microdon (three and one individuals, respectively) were included as outgroup taxa. Reithrodontomys sumichrasti and $R$. megalotis are members of the megalotis species group in the subgenus Aporodon, whereas $R$. microdon is a member of the subgenus Reithrodontomys. Thus, these taxa provide a paraphyletic outgroup for rooting the R. sumichrasti intraspecific phylogeography (E. Arellano and D. S. Rogers, unpublished data). In addition, we present sequence data for Peromyscus aztecus (Peromyscus aztecus evides) from the western end of the Sierra Madre del Sur (table 1) to supplement the data set of Sullivan et al. (1997).

\section{Data Collection}

DNA was extracted from approximately $10 \mathrm{mg}$ of liver tissue following Gustincich et al. (1991). Amplification of the cyt $\mathrm{b}$ gene was conducted using the following primers: L-14115: 5' -GATATGAAAAACCATCGTTG-3' and H-15915: 5'-CTGCAGTCATCTCCGGTTTACAAGAC-3'. Position numbers refer to the mitochondrial genome of Mus (Bibb et al. 1981). Amplified products were cleaned using polyethylene glycol (PEG) precipitation, and the entire gene was sequenced in four cyclesequencing reactions using the BigDye Kit (Applied Biosystems, Foster City, Calif.). In addition to the external PCR primers, the following internal primers were used for sequencing: L-14553: 5'-CTACCATGAGGACAAATATC-3' and H-14963: 5'-GGCAAATAGGAARTATCATT- $3^{\prime}$. Sequencing reactions were cleaned via gel filtration using CentriSep columns (Princeton Separations, Princeton, N.J.) and run on an ABI 377 automated sequencer using 4\% Long Ranger gels. This approach consistently produced reliable sequences of at least $600 \mathrm{bp}$; thus, approximately $90 \%$ of the gene was sequenced in both directions, with the remaining sequence being within $200 \mathrm{bp}$ of a sequencing primer. Only the first $700 \mathrm{bp}$ were sequenced for every individual, and each unique haplotype (as defined by the first $700 \mathrm{bp}$ ) was sequenced in its entirety. These sequences have been deposited in Genbank under accession numbers AF211894-AF211923.

\section{Phylogenetic Analyses}

Sequences were aligned using the automated contig assembly option in Sequencher (GeneCodes, Ann Arbor, Mich.); this was straightforward because no gaps were required. Phylogenetic analyses were conducted under both parsimony and likelihood optimality criteria using PAUP* (test version 4.0d61-d65; written by D. L. Swofford). Likelihood methods have the advantage of being explicitly model based and, therefore, are particularly well suited to statistical phylogenetic hypothesis testing (Huelsenbeck and Crandall 1997; Sullivan and Swofford 1997). However the computational demands of likelihood methods render impractical the ideal strategy of simultaneous optimization of all model parameters for each tree examined during a tree search. We, therefore, used an iterative-search strategy designed to circumvent the need of such simultaneous optimization (Swofford et al. 1996; Sullivan and Swofford 1997). This strategy entails an initial search under parsimony to identify a set of initial trees (i.e., trees that contain any long internal branches; Sullivan et al. 1996), evaluation of alternative maximum-likelihood (ML) models, estimation of the parameters of those models using the initial 
Table 1: Collection localities for the Reithrodontomys sumichrasti, outgroup, and Peromyscus aztecus samples examined here

\begin{tabular}{clll}
\hline Site & Region $^{\mathrm{a}}$ & \multicolumn{1}{c}{ Location } & $n$ \\
\hline Reithrodontomys sumichrasti: & & & \\
1 & SMOr & Mexico, Hidalgo, Rancho La Mojonara, 2,010 m & 6 \\
2 & SMOr & Mexico, Hidalgo, 10 km SW Tenango de Doria, 2,200 m & 2 \\
3 & OH & Mexico, Oaxaca, 1.5 km S Puerto de la Soledad, 2,600 m & 4 \\
4 & SMOr & Mexico, Veracruz, 6.5 km W Zacualtapan, 2,070 m & 3 \\
5 & CRH & Costa Rica, San Jose, Cascajal de Coronado, 1,650 m & 1 \\
6 & $\mathrm{CH}$ & Mexico, Chiapas, 13 km NE San Cristobal de las Casas & 4 \\
7 & SMdS & Mexico, Guerrero, Omiltemi & 5 \\
8 & SMOr & Mexico, Veracruz, Xometla & 2 \\
9 & OH & Mexico, Oaxaca, 27.5 km W Orizaba & 2 \\
10 & SMdS & Mexico, Oaxaca, 4.8 km S Suchixtepec & 2 \\
11 & CT & Mexico, Michoacan, 6.2 km W Mil Cumbres & 1 \\
12 & CT & Mexico, Michoacan, 3.2 km E Opopeo & 3 \\
13 & SMdS & Mexico, Guerrero, 10.2 km SW Filo de Caballo & 2 \\
14 & SMOr & Mexico, Hidalgo, 5.0 km N Zacoultipan & 1 \\
15 & SMOr & Mexico, Hidalgo, 0.5 km N Molango & 2 \\
16 & CT & Mexico, Michoacan, 1.9 km S Villa Escalante & 1 \\
17 & CH & Mexico, Chiapas, Rayon & 1 \\
18 & CH & Mexico, Chiapas & 1 \\
Reithrodontomys megalotis & $\ldots$ & Mexico, D.F., Desierto de los Leones & 3 \\
Reithrodontomys microdon & $\ldots$ & Mexico, Chiapas, 13 km NE San Cristobal de las Casas & 1 \\
Peromyscus aztecus evides & $\ldots$ & Mexico, Guerrero, Omiltemi & 2 \\
\hline
\end{tabular}

${ }^{a}$ Region abbreviations are as follows: $\mathrm{SMOr}=$ Sierra Madre Oriental; $\mathrm{OH}=$ Oaxacan Highlands; $\mathrm{CRH}=\mathrm{Costa}$ Rican Highlands; $\mathrm{CH}=$ Chiapan Highlands; SMdS = Sierra Madre del Sur; CT = Cordillera Transvolcanica. Together, the CRH and $\mathrm{CH}$ for the region TIH (trans-isthmian highlands).

trees, and subsequent tree search(es) under the likelihood criterion, using an objectively determined and fully defined model. The initial parsimony searches (heuristic searches with 100 replicate random addition sequences and tree bisection-reconnection branch swapping $[\mathrm{TBR}]$ ) were conducted using equal weights. Because use of an appropriate likelihood model can be crucial to statistical tests of phylogenetic hypotheses (e.g., Sullivan and Swofford 1997; Zhang 1999), the relative fit of 16 alternative models of sequence evolution was assessed across the six parsimony trees by means of likelihood-ratio tests. Yang et al. (1995), provided a justification of use of the $\chi^{2}$-approximation to the null distribution, but Whelan and Goldman (1999) indicated conditions under which the $\chi^{2}$ distribution differs significantly from the distribution generated by simulation under the null hypothesis. These 16 models are the same as Sullivan et al. (1997) used in their examination of the phylogeography of the P. aztecus/Peromyscus hylocetes complex, and a comparison of the explicit assumptions of these models is provided by Sullivan and Swofford (1997).

Subsequent heuristic searches (10 random addition sequences, TBR branch swapping) were then conducted under likelihood with the fully defined, best-fit model. Because the presence of multiple zero-length internal branches (which were also detected in the parsimony analyses; see below) caused likelihood searches to bog down in evaluation of alternative resolutions of polytomies (zero-length internal branches), ML analyses were conducted on a reduced data set from which four haplotypes were omitted: cwk1009, fac1112, jmv1571, jmv1640. These were chosen for omission because the parsimony analyses suggested that inclusion of these haplotypes results in a large number of zero-length internal branches. Furthermore, a strict consensus of the two most parsimonious (MP) trees for the reduced data set is identical to the pruned strict consensus of the MP trees for the full data set (not shown).

Although the likelihood-ratio tests used above provide an evaluation of the relative fit of several alternative nested models, and thereby provide an objective means of model choice, they can provide no indication of the adequacy of the chosen model. That is, simply because the chosen model fits the data better than any of the alternatives examined, it may nevertheless fit the data poorly. Therefore, we examined the adequacy of the chosen model following Goldman (1993). Under this approach, goodness-of-fit can be assessed by the difference between the unconstrained likelihood (the multinomial likelihood) and the likelihood of the ML tree under the model being examined. The null 
hypothesis is that this difference is no greater than would be expected by chance were there a perfect fit between model and data; the null distribution was generated via simulation under the candidate model, with parameters of that estimated from the original data.

Nodal support was estimated by bootstrap analysis (Felsenstein 1985). For parsimony bootstrap analyses, we used 500 replicates, each of which was subjected to a full heuristic search (i.e., with TBR branch swapping). For likelihood bootstrap analyses, MAXTREES was set to one in the analyses of each of the replicates, and TBR branch swapping was allowed to continue to completion. This approach may be viewed as intermediate between more conventional bootstrap analyses and the FastBoot approach, which uses random input orders to build a tree for each pseudoreplicate data set via stepwise addition (no branch swapping is conducted). Although the behavior of the approximate FastBoot method is not thoroughly understood, Felsenstein (1993) has suggested that the error measured by the resampling procedure is likely to be much greater than that associated with branch swapping. In addition, there is some indication that the FastBoot procedure is conservative in its estimation of nodal support, at least in parsimony analyses (Mort et al. 2000). Thus, nodes that receive strong support in our intermediate ML bootstrap analyses would likely be more strongly supported were the analyses of pseudoreplicate data sets to have saved more than a single tree on which to swap.

\section{Tests of Alternative Hypotheses}

Statistical tests of phylogeographic hypotheses were conducted using three methods. Kishino-Hasegawa-Templeton (KHT tests; Templeton 1983; Kishino and Hasegawa 1989) and parametric bootstrap tests (Efron and Tibshirani 1993; Huelsenbeck et al. 1996) were used to test phylogeographic congruence (i.e., the concerted-response hypothesis), and a randomization test of reconciled tree maps (Page 1994) was used to assess the significance of shared history between the two groups (i.e., with the independent-response hypothesis as the null). Both KHT and parametric bootstrap tests were conducted under both parsimony and likelihood, with the likelihood tests conducted using the reduced data set. These two tests employ the same test statistic: the difference in optimality score (either tree length or ln likelihood) between the optimal tree and the tree constrained to fit the hypothesis under examination. The null hypothesis is identical for both tests; there is no greater incongruence between the phylogeographic patterns observed in R. sumichrasti and the P. aztecus/P. hylocetes complex than can be attributed to uncertainty in phylogenetic estimation. The difference between these tests lies in the null distribution used to derive $P$ values. In the
KHT test, the null distribution is generated directly from the data; under the hypothesis that there is no difference between the two trees being compared, single-site differences in optimality score are expected to be normally distributed (Kishino and Hasegawa 1989) and a simple $t$-test may be used. For the parametric-bootstrap test, the null distribution is generated via simulation under the null hypothesis and is therefore independent of the data under examination. Reciprocal comparisons were conducted for each of these tests.

The third test entailed tree mapping (Page 1994). This method was designed to analyze cospeciation between parasite and host lineages; however, it can also be used to assess shared history between codistributed groups. TREEMAP (version 1.0; Page 1994) was used to estimate the degree of shared history between the two complexes; this is measured by the inferred number of covicariance events in the reconciliation. For this test, the null hypothesis is that there is no greater covicariance than would be expected at random for two groups with completely independent histories. The significance of the number of covicariance events inferred from the data was assessed by generating comparisons between 5,000 pairs of random trees. In each of these comparisons, the phylogeographies of both species complexes were randomized.

\section{Results}

\section{Phylogenetic Analyses}

Based on the first $700 \mathrm{bp}$ of the cyt b gene, we identified 30 unique haplotypes within the sample of 43 individuals of Reithrodontomys sumichrasti examined. Uncorrected divergence levels ( $p$ distances) among these haplotypes (for the $1,130 \mathrm{bp}$ ) within $R$. sumichrasti ranged from $0 \%$ to $7.7 \%$. Between R. sumichrasti and the outgroup taxa, divergences ranged from $6.9 \%$ to $8.7 \%$ for comparisons with Reithrodontomys megalotis, and from $12.9 \%$ to $14.7 \%$ for comparisons with Reithrodontomys microdon. Including outgroups, 260 sites were observed to vary; 168 of these were parsimony informative. The distribution of variation among codon positions is as expected for a coding gene; 48 first codon-position sites were observed to vary (23 parsimony-informative sites), eight second codon-position sites were observed to vary (two parsimony-informative sites), and 204 third codon-position sites were observed to vary (138 parsimony-informative sites). This distribution of variation, as well as the absence of stop codons, frameshift substitutions, and radical amino-acid substitutions suggests that these are in fact mtDNA sequences rather than those of a nuclear pseudogene.

Initial parsimony analysis with equal weights resulted in six equally parsimonious trees (451 steps; consistency 
index of 0.547, excluding uninformative sites; rescaled consistency index of 0.5545 ); the ingroup topologies differed primarily in the resolution of near zero-length internal branches. Two of the topologies are identical to that shown in figure 3 , and the remaining four differed in that $\mathrm{SMdS}_{\mathrm{W}}$ samples formed a paraphyletic group. In general, there are several well-supported nodes and a few nodes with low bootstrap support. The samples from south and east of the Isthmus of Tehuantepec form a well-supported clade (labeled TIH for the trans-Isthmian highlands in figs. 1,3 , and 4), as do all the samples from north and west of the isthmus (fig. 3). Within this latter clade, samples from the Sierra Madre Oriental (SMOr) form a well-supported group, as do the remaining samples from central Mexico. This last clade (the $\mathrm{OH} / \mathrm{CT} / \mathrm{SMdS}$ clade) includes the samples from the Oaxacan Highlands $(\mathrm{OH})$ and the Cordillera Transvolcanica (CT), each of which themselves represent a well-supported monophyletic assemblage of haplotypes, as well as the samples from the entire Sierra Madre del Sur (SMdS). However, the samples from the Sierra Madre del Sur do not form a monophyletic group. The small sample $(n=2)$ from the eastern end of this

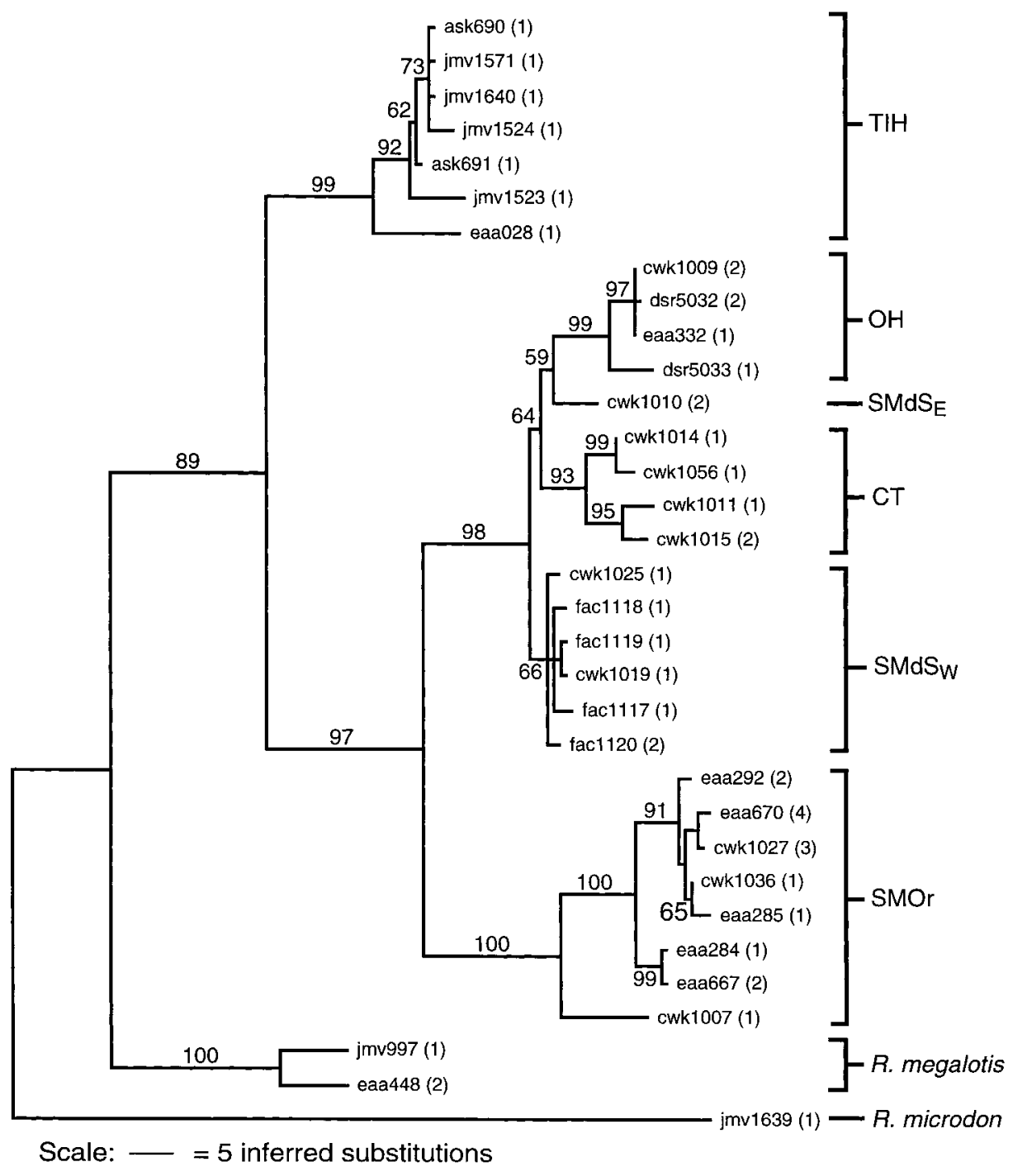

Figure 3: One of six equally parsimonious trees found using equal weights. This tree has a length of 451 steps, a consistency index of 0.547 (excluding uninformative sites), and a rescaled consistency index of 0.5545 . Numbers in parentheses following sequence labels represent the number of individuals with the identical haplotype. Numbers associated with each node represent bootstrap values (500 replicates). Nodes without bootstrap values received $<50 \%$ bootstrap support. This tree differs from one of the other MP trees only by alternative resolutions of zero-length internal branches and differs from the other four MP trees in that the haplotypes from $\mathrm{SMdS}_{\mathrm{w}}$ are paraphyletic in those. Branch lengths inferred using ACCTRAN. 


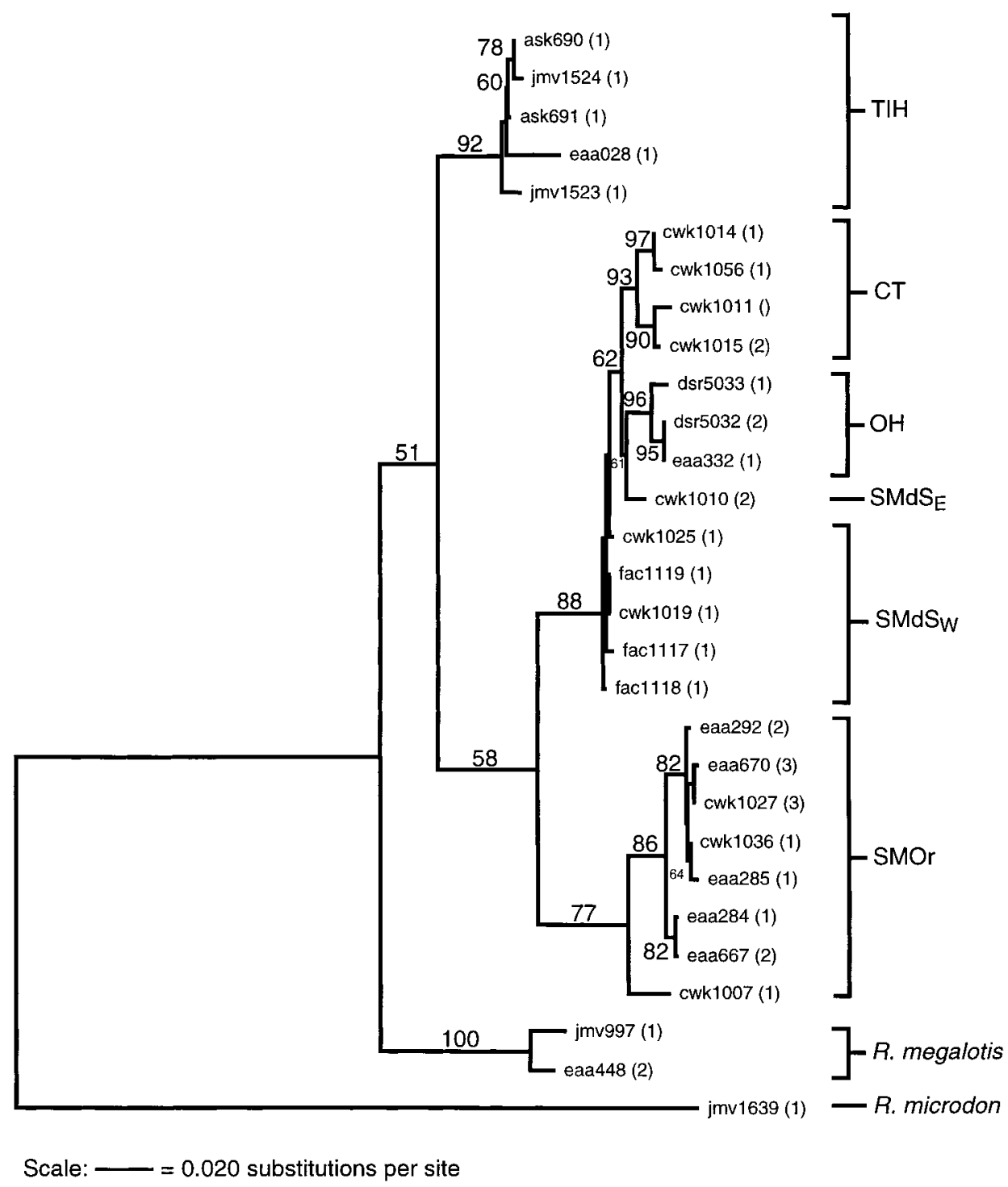

Figure 4: One of three ML trees found using the objectively determined GTR $+\mathrm{I}+\Gamma$ model of sequence evolution (see text for justification) estimated from the reduced data set. This tree has a likelihood score (ln likelihood) of -3679.29649 , and differs from the other ML trees only by alternative resolutions of zero-length internal branches. Numbers associated with each node represent estimates of bootstrap values (100 replicates). Each replicate was analyzed with MAXTREES set to one and with full TBR branch swapping on stepwise addition trees generated with random addition sequeunce.

mountain range, $\mathrm{SMdS}_{\mathrm{E}}$, is weakly supported as sister group to the Oaxacan Highland samples. In some of the MP trees (not shown), the samples from the western end of the Sierra Madre del Sur, $\mathrm{SMdS}_{\mathrm{w}}$, are paraphyletic and form a grade at the base of the OH/CT/SMdS clade. In addition, there is moderate support for a sister-group relationship between the samples from the Cordillera Transvolcanica and the $\mathrm{SMdS}_{\mathrm{E}} / \mathrm{OH}$ clade (fig. 3).

Results of the ML analysis are similar (fig. 4). The GTR $+\mathrm{I}+\Gamma$ model of sequence evolution fit the data sig- nificantly better than any of its special cases we examined $(P<.01)$ and actually appears to fit the data quite well (fig. 5). The observed difference between the unconstrained (multinomial) likelihood and the likelihood score of the ML tree under the model falls in the middle of the distribution of simulated data generated under the null hypothesis of a perfect fit between model and data (fig. 5). Thus, the otherwise untested assumptions of the GTR $+\mathrm{I}+\Gamma$ model (i.e., stationarity and reversibility of process, independence of sites) are probably not violated 


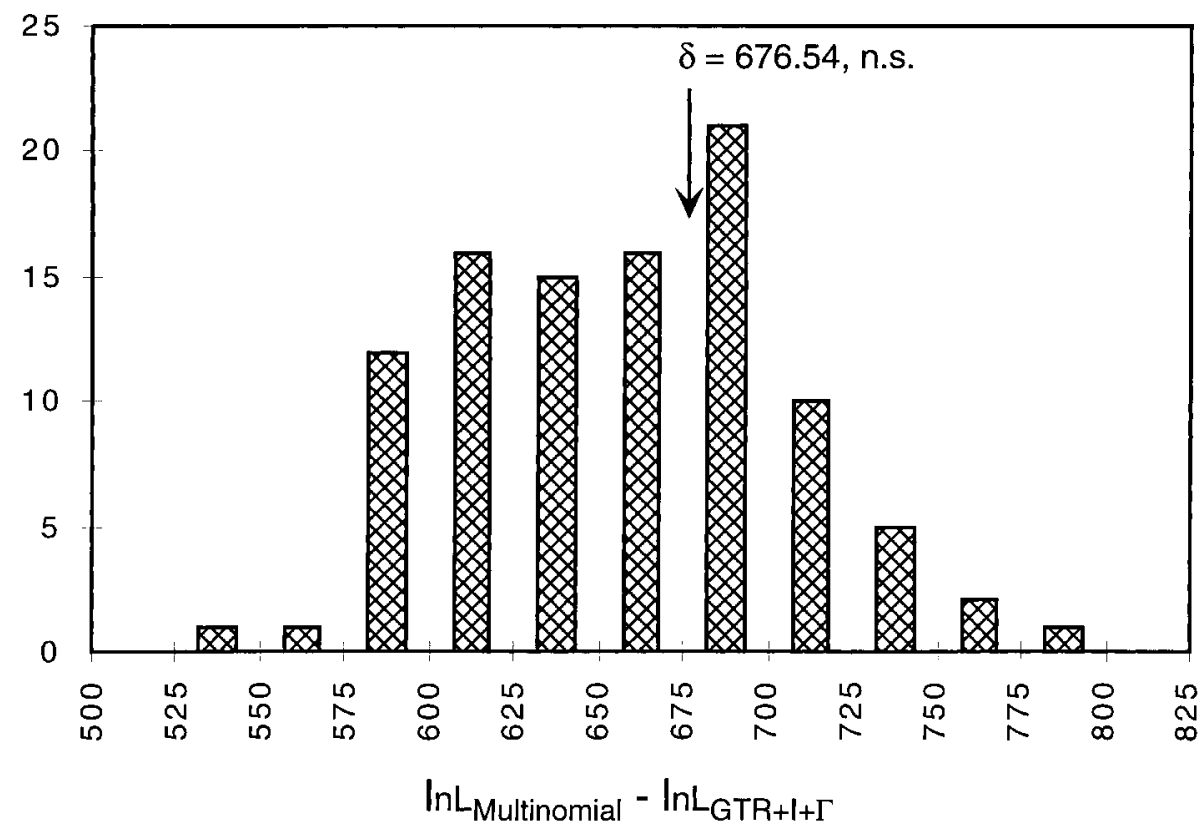

Figure 5: Parametric bootstrap test of the goodness-of-fit between the GTR $+\mathrm{I}+\Gamma$ model of sequence evolution and the Reithrodontomys sumichrasti cyt $b$ data. One hundred replicate data sets were simulated under the GTR $+\mathrm{I}+\Gamma$ model, and the distribution of the difference between unconstrained (multinomial) $\ln$ likelihoods and ln likelihood of the ML tree under the model is shown. The difference observed in the real data falls in the middle of the distribution generated under the null hypothesis of a perfect match between model and data; the GTR $+\mathrm{I}+\Gamma$ model fits the data quite well.

in a manner that will alter the statistical tests of alternative hypotheses ("Hypothesis Tests"). The heuristic searches using the fully defined GTR $+\mathrm{I}+\Gamma$ model resulted in three ML trees, which differ only by resolution of zero-length internal branches. However, in all of the three ML trees, the $\mathrm{SMdS}_{\mathrm{W}}$ samples form a paraphyletic group at the base of the $\mathrm{OH} / \mathrm{CT} / \mathrm{SMdS}$ clade. In general, the likelihood bootstrap values are lower than corresponding parsimony bootstrap values; however, there are several nodes that are strongly supported (fig. 4).

\section{Hypothesis Tests}

There are both striking similarities and strong differences between the phylogeographic relationships of $R$. sumichrasti and the Peromyscus aztecus/Peromyscus hylocetes complex (Sullivan et al. 1997). The first similarity is that identical evolutionarily significant units (ESUs; e.g., Moritz 1995) are present in both complexes; for the most part separate mountain ranges represent monophyletic assemblages of haplotypes (figs. 3, 4). The exception to this is that the samples of $R$. sumichrasti from the western end of the Sierra Madre del Sur, $\mathrm{SMdS}_{\mathrm{W}}$, may not represent a monophyletic group. Second, the Isthmus of Tehuantepec is a strong phylogeographic barrier in both complexes, with the deepest divergences within each of these groups corresponding geographically to the Isthmus.

Incongruence between the two phylogeographies lies in the phylogenetic position of the samples from the Sierra Madre Oriental and the Cordillera Transvolcanica. In $R$. sumichrasti, the sister clade to the $\mathrm{OH} / \mathrm{SMdS}_{\mathrm{E}}$ clade is the Cordillera Transvolcanica clade (figs. 3, 4) rather than the Sierra Madre Oriental clade, as was the case in the P. aztecus phylogeography (fig. 2; Sullivan et al. 1997). Furthermore, this incongruence is significant. The best $R$. sumichrasti tree constrained to fit the $P$. aztecus/ $P$. hylocetes complex phylogeography (fig. 2) is significantly worse than the best unconstrained $R$. sumichrasti trees (table 2 ; fig. 6). This is true across most of the tests we employed, including reciprocal tests in which the $P$. aztecus/P. hylocetes complex data were forced to fit the $R$. sumichrasti tree. The exception was that the parsimony parametric-bootstrap test resulted in $P=.108$; all the other tests (ML parametric bootstrap, ML KHT test, and parsimony KHT test) provided strong evidence that the two phylogeographies are significantly incongruent ( $P$ values ranged from .005 to .097; table 2; fig. 6).

The tree-mapping analysis indicated that these phylogeographies can be reconciled by a single duplication event, and that there have been four covicariance events shared by these two groups (fig. 7). Based on comparisons 
Table 2: Reciprocal statistical tests of phylogeographic congruence between Reithrodontomys sumichrasti and the Peromyscus aztecus/Peromyscus hylocetes complex

\begin{tabular}{|c|c|c|c|c|}
\hline \multirow{2}{*}{$\begin{array}{l}\text { Optimality } \\
\text { criterion } \\
\text { and test }\end{array}$} & \multicolumn{2}{|c|}{ R. sumichrasti $i^{\mathrm{a}}$} & \multicolumn{2}{|c|}{$\begin{array}{l}\text { P. aztecus/ } \\
\text { P. hylocetes }\end{array}$} \\
\hline & $\delta$ & $P$ & $\delta$ & $P$ \\
\hline \multicolumn{5}{|l|}{ Parsimony: } \\
\hline KHT & 12 & .005 & 6 & .080 \\
\hline PBT & 12 & .108 & 6 & .078 \\
\hline \multicolumn{5}{|l|}{ Likelihood: } \\
\hline KHT & 12.460 & .097 & 6.830 & .027 \\
\hline PBT & 12.460 & $\ll .01$ & 6.830 & $\ll .01$ \\
\hline
\end{tabular}

Note: Abbreviations are as follows: KHT = Kishino-Hasegawa-Templeton test test; $\mathrm{PBT}=$ parametric bootstrap test. Data from Sullivan et al. 1997.

${ }^{a}$ Reithrodontomys sumichrasti data forced to fit Peromyscus aztecus/Peromyscus hylocetes phylogeography.

${ }^{\mathrm{b}}$ Peromyscus aztecus/P. hylocetes data forced to fit R. sumichrasti phylogeography.

of random trees, this degree of covicariance would be expected to occur at a frequency of 0.02 , if there were no commonality between the two groups (i.e., if ESUs within each group were partitioning at random geographically, with respect to each other). Thus, we can reject the null hypothesis of independent response $(P=.02)$; there is greater similarity between the phylogeographies of the two complexes than expected at random.

\section{Discussion}

\section{Phylogeographic Congruence}

Assessment of the degree to which codistributed taxa have congruent phylogeographies is critical because, in instances where congruence is detected, shared history has led to similar partitioning of genetic variation in the taxa being compared. This enables the prediction of geographic patterns of genetic variation for other codistributed taxa that have yet to be examined. In this study, for both Reithrodontomys sumichrasti and the Peromyscus aztecus/Peromyscus hylocetes complex, populations from separate mountain ranges generally represent monophyletic assemblages of haplotypes. Thus, identical ESUs are identifiable in both complexes, and this provides the prediction that this will also be the case for other groups similarly restricted to these disjunct mid- to high-elevation forests. This predictive aspect of comparative phylogeography provides a basis for the application of a systemwide approach to the conservation of genetic diversity in this region (Avise 1992, 1998).

The deepest phylogeographic divergence in R. sumichrasti is the separation of the trans-Isthmian populations (subspecies R. sumichrasti australis, R. sumichrasti dorsalis, R. sumichrasti modestus, and R. sumichrasti vulcanius) from those in central and southern Mexico (subspecies R. sumichrasti luteolus, R. sumichrasti nerteus, and R. sumichrasti sumichrasti). This was also the deepest phylogeographic split that Sullivan et al. (1997) observed in the P. aztecus/ $P$. hylocetes complex. The divergence level represented by this split is quite high in both complexes; uncorrected sequence divergence levels range from $5.6 \%$ to $7.7 \%$ among $R$. sumichrasti samples and from $6.9 \%$ to $8.9 \%$ in the $P$. aztecus/P. hylocetes complex samples. Unfortunately, neither of these data sets fits the molecular clock hypothesis. Because Felsenstein's (1988) likelihood ratio test of the molecular clock allows for use of models that are tailored to fit the data set at hand, we consider it to be the most appropriate of the alternative tests of the clock hypothesis. Application of this test, using the best-fit model of sequence evolution in each case, results in rejection of the clock: $\delta_{24}=43.374(P<.01)$ for $R$. sumichrasti; $\delta_{13}=$ $33.377(P<.005)$ for the $P$. aztecus/P. hylocetes complex. This prohibits us from examining the temporal congruence of this common phylogeographic split in the two groups and prohibits use of the clock to place an absolute divergence time estimate on the split. However the data are consistent with relatively deep divergences, perhaps relating to the most recent marine transgressions across the Isthmus of Tehuantepec in the late Pliocene (e.g., Maldonado-Koerdell 1964).

The taxonomic level that should be attributed to this split in R. sumichrasti is not entirely clear. Although current taxonomy unites these segments as conspecific, there has been some suggestion that the trans-Isthmian populations represent a distinct species. For example, Engstrom et al. (1981) noted strong differentiation in the karyotypes between a small sample of mice from Chiapas and Michoacan, Mexico, and suggested the possibility that R. sumichrasti as currently delimited is a composite of two species. The high levels of differentiation seen here in the cyt b sequences is concordant with this suggestion, although certainly morphological and molecular data from nuclear markers should be brought to bear. The disjunct nature of the segments will prohibit the application of the criterion of reproductive isolation that has traditionally been applied to rodent systematics (i.e., Rennert and Kilpatrick 1986), and a genealogical concordance approach to species limits (i.e., Baum and Shaw 1995) will be more applicable. We predict that the trans-Isthmian differentiation observed here and by Engstrom et al. (1981) will persist across other character sets, and application of the concordance concept will require the recognition of these populations as specifically distinct. 

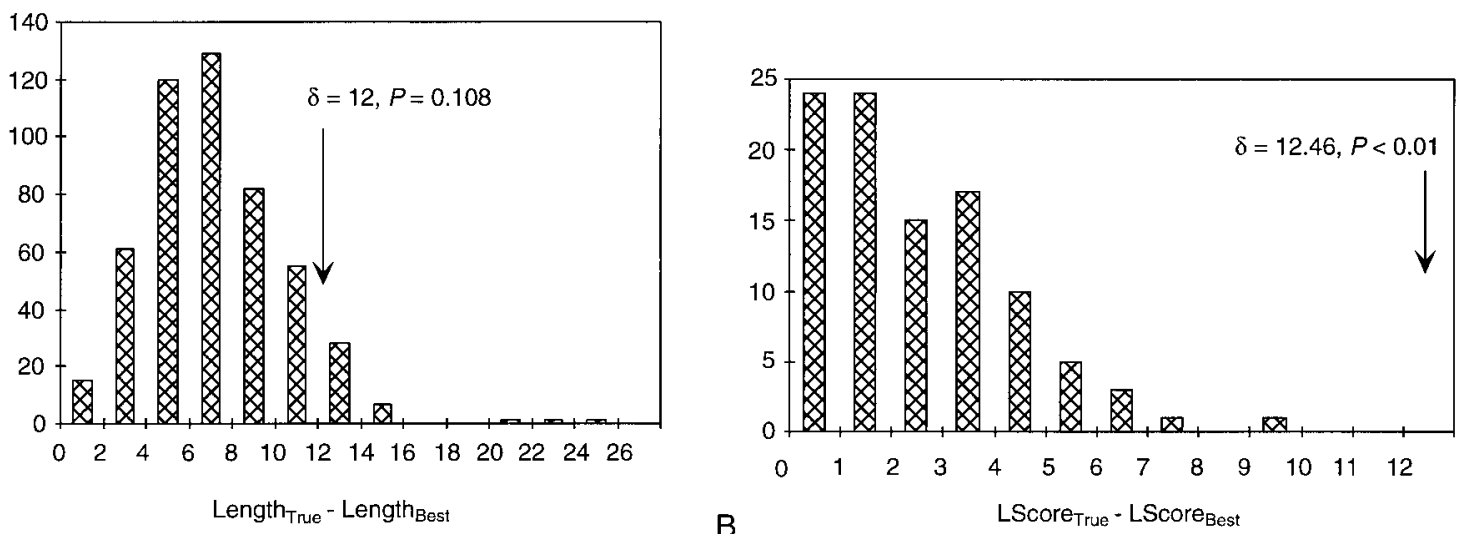

\section{A}
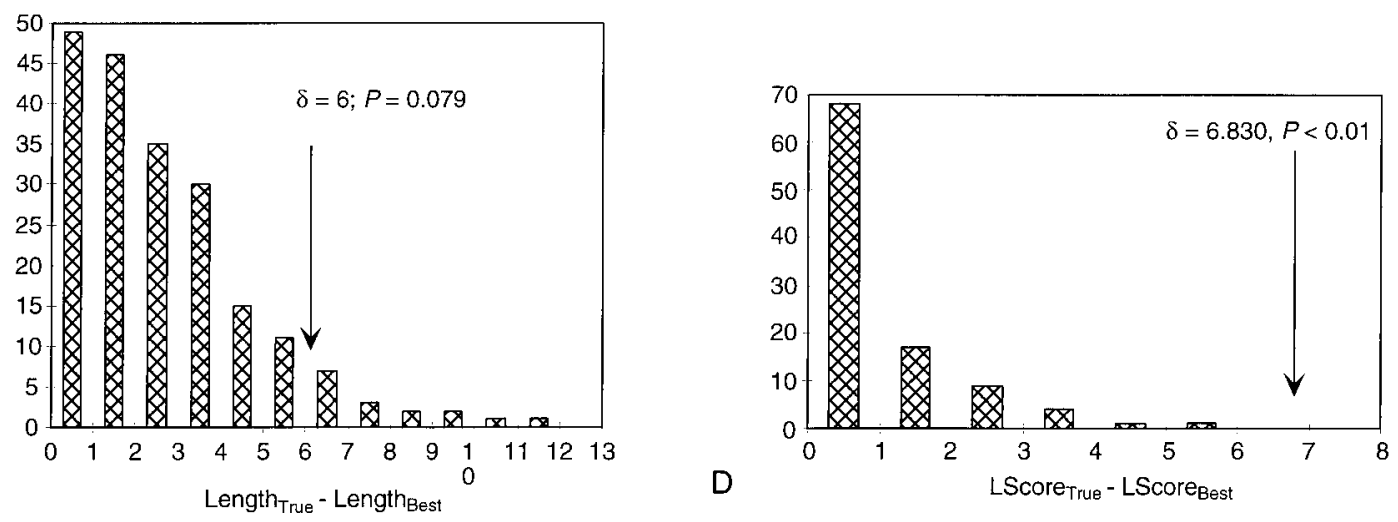

Figure 6: Parametric bootstrap tests of phylogeographic congruence. $A, B$, Tests conducted under parsimony and likelihood, respectively, with the Reithrodontomys sumichrasti data constrained to fit the area relationships inferred from the Peromyscus aztecus/Peromyscus hylocetes complex (fig. 2). $C, D$, Tests conducted under parsimony and likelihood, respectively, with the P. aztecus/P. hylocetes data (Sullivan et al. 1997) constrained to fit the area relationships inferred from $R$. sumichrasti. Two hundred fifty replicate data sets were simulated for each of the parsimony tests $(A, C)$, and 100 replicates were simulated for the likelihood tests $(B, D)$. These reciprocal tests reject the hypothesis that the observed incongruence between the phylogeographies of the two groups is attributable to uncertainty in phylogenetic estimation.

\section{Phylogeographic Hypotheses}

In part owing to the relevance of comparative phylogeography to conservation and in part owing to the need to bridge ecological and historical biogeography, numerous studies have taken the lead from Avise (1992) in attempting to assess comparative phylogeography on a regional scale (e.g., Riddle 1995; Zink 1996; Bermingham and Martin 1998; Taberlèt et al. 1998). In searching for congruence among phylogeographies of codistributed forms, however, many of these studies have relied on visual inspection of phylogeographic estimates to infer common patterns. This clearly is an inadequate assessment of congruence (e.g., Hillis 1995). Tools provided by Page $(1988,1994)$ provide historical biogeographers with the statistical approach needed to assess similarity between area relationships, and indeed a few studies have employed such a statistical approach (e.g., Bermingham and Martin 1998). In addition, with the increasing attention being focused on statistical phylogenetics (e.g., Huelsenbeck and Crandall 1997), comparative phylogeographers now have the ability to incorporate phylogenetic uncertainty in tests of biogeographic hypotheses. Thus, through the use of tests such as the parametric bootstrap, instances of real incongruence can be discerned from instances in which apparent differences are not statistically significant, but rather arise from uncertainty in phylogenetic estimation.

In the current example, we are left with conflicting results, depending on which hypothesis is taken as the null. If the classical concerted-response hypothesis is taken as the null, the results of both the parametric bootstrap (table 2; fig. 6) and KHT tests (table 2) indicate that there is significant incongruence between the phylogeographies of these two groups. This incongruence can be localized to the difference in placement of the Sierra Madre Oriental 


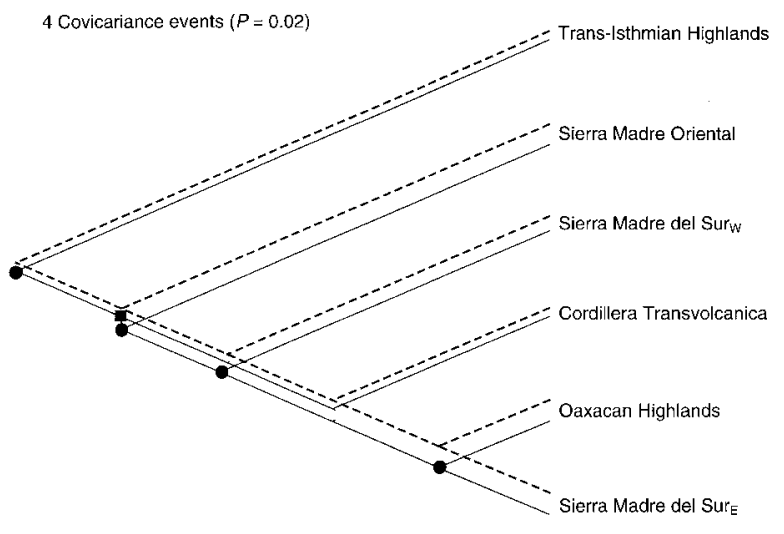

Figure 7: Tree mapping of the estimates of area relationships inferred from Reithrodontomys sumichrasti and the Peromyscus aztecus/Peromyscus hylocetes complex. The solid tree represents the inference based on $R$. sumichrasti, whereas the dashed tree represents the estimates based on the P. aztecus/P. hylocetes complex. A single duplication (square) and four covicariance events (circles) are required to reconcile these hypotheses of area relationships; this represents a greater degree of shared history than would be predicted by the independent-response hypothesis $(P=.02)$.

ESU. The SMOr samples were sister to the $\mathrm{SMdS}_{\mathrm{E}} / \mathrm{OH}$ clade, with the CT clade (P. hylocetes) sister to these in the Peromyscus analyses (fig. 2; Sullivan et al. 1997), whereas the SMOr populations are sister to the entire central Mexican clade in the Reithrodontomys analyses (figs. 3, 4). Thus, we can reject the concerted-response hypothesis as a complete description of the distributional association between these two taxa. With the independent-response hypothesis taken as the null, reconstruction of the historical association of these two taxa using TREEMAP (Page 1994) required four covicariance events to reconcile the area relationships inferred from the two taxa (fig. 7). This is a greater degree of shared history (i.e., greater covicariance) than would be expected under random associations of lineages for the two groups. Thus, we can also reject the independent-response hypothesis. In this case, the truth appears to lie between these two competing paradigms; our analyses indicate that there has been some correlation in the responses of these two taxa to the past climatic and geologic events, but the responses have not been entirely coupled. The two groups involved belong to very closely related genera (Peromyscus and Reithrodontomys) and have very similar ecologies and life histories. It will be interesting to see to what degree the mixed patterns observed here are also observed in phylogeographic comparisons of the codistributed taxa in the Mesoamerican highland forests with different ecologies and life histories.
Use of Reconciled Trees in Comparative Biogeography

The manner in which we used TREEMAP to assess the degree of shared history between $R$. sumichrasti and the $P$. aztecus/P. hylocetes complex deviates somewhat from typical uses of tree reconciliation (i.e., as suggested by Andersson 1996). For example Bermingham and Martin (1998) used tree reconciliation to assess the commonality in patterns of dispersal of several species of freshwater fishes across the Panamanian land bridge. As is typically done, these authors assessed shared history by use of COMPONENT analysis (Page 1993) to derive a general area cladogram from the single-taxon area cladograms, and then used tree reconciliation between this general area cladogram and each of the single-taxon area cladograms used in its construction. Thus, the general area cladogram used in their examination of shared history was not independent of the single-taxon area relationships because each single-taxon hypothesis under examination was used in the construction of the general area cladogram. Our reconciliation of one single-taxon hypothesis of area relationships directly against the other has two advantages over such an approach. First, the approach we use eliminates this problem of nonindependence. Second, when comparing two hypotheses of area relationships, any composite estimate will be intermediate between the original single-taxon area cladograms. Therefore, an indication of more commonality (in our case covicariance) than expected at random will be a much more rigorous statement of shared history, if the individual hypotheses of area relationships are tested directly against one another than would be the case were the single-taxon trees tested against a composite estimate that must necessarily be intermediate.

Nevertheless, one weakness of either approach is that both ignore uncertainty in estimation of single-species phylogeographies. That is, tree reconciliation treats each species phylogeography as if it were inferred without error. This may be an issue in the current data set in that the association of the $\mathrm{SMdS}_{\mathrm{E}}$ with the $\mathrm{OH}$ samples (and the vicariance event that this implies) is not particularly well supported (figs. 3, 4) nor is the $\mathrm{SMdS}_{\mathrm{E}}$ region particularly well sampled. Running the TREEMAP analyses with these two regions treated as a single area results in three covicariance events, and the probability of observing this reduced degree of shared history increases correspondingly $(P=.107)$. However this result is difficult to interpret because much of the decrease in significance is attributable simply to loss of statistical power because there are fewer regions to reconcile. 


\section{Conclusions}

This study has demonstrated that the competing paradigms of concerted versus independent responses of codistributed forms may represent a false dichotomy. Others have suggested such a pattern should be expected (Cracraft 1994; Andersson 1996); however, there were no statistical tests of the opposing hypotheses in those studies. We have demonstrated statistically that the best explanation of the responses of currently codistributed species to past climatic events is a combination of the existing paradigms or a mixed-response hypothesis. While it is entirely likely that this is a general pattern that will persist across examinations of concerted versus independent responses to other geological and climatic events (e.g., Bermingham and Martin 1998), the mixed-response model presents its own difficulties. Perhaps the greatest of these is that the mixedresponse hypothesis cannot be used to make explicit testable predictions. At this point, the best that can be predicted under the mixed-response hypothesis is that perfect phylogeographic congruence among codistributed forms will not occur, yet codistributed taxa will share more common history than would be predicted under a completely independent-response scenario. Thus, both the concerted- and independent-response hypotheses will continue to play a pivotal role in historical biogeography by serving as testable null models.

\section{Acknowledgments}

We wish to thank F. A. Cervantes, F. X. González, Y. Hortelano, C. W. Kilpatrick, and J. Martinez either for generously donating samples or for assistance in collection of samples used here. J. Demboski, S. Emerson, M. Fishbein, B. Riddle, and an anonymous reviewer provided very useful comments on the manuscript. J. Good provided laboratory assistance. We also thank D. L. Swofford for discussions of statistical issues, for writing the software used for simulations, and for providing access to test versions of PAUP*. This research was supported by start-up funds provided to J.S. from the University of Idaho and a seed grant from the University of Idaho Research Foundation (to J.S.), National Institutes of Health (NIH) Grant 1R15.GM46016-01, Professional Development Grants, Department of Zoology and Monte L. Bean Life Science Museum, Brigham Young University (to D.S.R.), the Consejo Nacional de Ciencia y Tecnología, the Fullbright Foundation, and the Theodore Roosevelt Memorial Fund of the American Museum of Natural History (to E.A.). In addition, some of the equipment used for this study was provided by the NIH (DHHS/NIH RR11833) to the University of Idaho Department of Biological Sciences. We would like to honor the memory of Danny Harris, whose friendship greatly facilitated this collaboration and who is sorely missed.

\section{Literature Cited}

Andersson, L. 1996. An ontological dilemma: epistemology and methodology of historical biogeography. Journal of Biogeography 23:269-277.

Avise, J. C. 1992. Molecular population structure and the biogeographic history of a regional fauna: a case history with lessons for conservation biology. Oikos 63:62-76. - 1998. The history and purview of phylogeography: a personal reflection. Molecular Ecology 7:317-379.

Baum, D. A., and K. L. Shaw. 1995. Genealogical perspectives on the species problem. Pages 289-302 in $\mathrm{P}$. C. Hoch and A. G. Stephenson, eds. Experimental and molecular approaches to plant biosystematics. Monographs in Systematic Botany. Vol. 53. Missouri Botanical Garden, St. Louis.

Bennett, K. D. 1990. Milankovitch cycles and their effects on species in ecological and evolutionary time. Paleobiology 16:11-21.

Bermingham, E., and A. P. Martin. 1998. Comparative mtDNA phylogeography of Neotropical freshwater fishes: testing shared history to infer the evolutionary landscape of lower Middle America. Molecular Ecology 7:499-517.

Bibb, M. J., R. A. van Etten, C. T. Wight, M. W. Wallberg, and D. A. Clayton. 1981. Sequence and gene organization of mouse mitochondrial DNA. Cell 26:167-180.

Brooks, D. R. 1981. Hennig's parasitological method: a proposed solution. Systematic Zoology 30:229-249.

Brown, J. H., and M. V. Lomolino. 1998. Biogeography. Sinauer. Sunderland, Mass.

Carleton, M. D. 1979. Taxonomic status and relationships of Peromyscus boylii from El Salvador. Journal of Mammalogy 60:280-296.

Cracraft, J. 1994. Species diversity, biogeography, and the evolution of biotas. American Zoologist 34:33-47.

Efron, B., and R. J. Tibshirani. 1993. An introduction to the bootstrap. Chapman \& Hall, New York.

Engstrom, M. D., R. C. Dowler, D. S. Rogers, D. J. Schmidly, and J. W. Bickham. 1981. Chromosomal variation within four species of harvest mice (Reithrodontomys). Journal of Mammalogy 62:159-164.

Fa, J. E., and L. Morales. 1993. Patterns of mammalian diversity in Mexico. Pages 319-361 in T. P. Ramamorthy, R. Bye, A. Lot, and J. Fa, eds. Biological diversity of Mexico: origins and distribution. Oxford University Press, New York.

FAUNMAP Working Group. 1996. Spatial response of mammals to late quaternary environmental fluctuations. Science (Washington, D.C.) 272:1601-1606. 
Felsenstein, J. 1985. Confidence limits on phylogeny: an approach using the bootstrap. Evolution 39:783-791. 1988. Phylogenies from molecular sequences: inference and reliability. Annual Review of Genetics 22: 521-565.

1993. PHYLIP, version 3.5c. Computer program distributed by the author. University of Washington, Seattle.

Flores-Villela, O., and P. Gerez. 1994. Biodiversidad y conservación en México: vertebrados, vegetación y uso del suelo. 2d ed. Comisión Nacional Para el Conocimiento y Uso de la Biodiversidad y Universidad Nacional Autónoma de México, México.

Goldman, N. 1993. Statistical tests of models of DNA substitution. Journal of Molecular Evolution 36:182-198.

Graham, R. W. 1986. Response of mammalian communities to environmental changes during the late quaternary. Pages 300-313 in J. M. Diamond and T. J. Case, eds. Community ecology. Harper \& Row, New York.

Gustincich, S., G. Manfioletti, G. Del Sal, C. Schneider, and P. Carnichi. 1991. A fast method for high quality genomic DNA extraction from whole human blood. BioTechniques 11:298-301.

Hillis, D. M. 1995. Approaches for assessing phylogenetic accuracy. Systematic Biology 44:3-16.

Huelsenbeck, J. P., and K. A. Crandall. 1997. Phylogeny estimation and hypothesis testing using maximum likelihood. Annual Review of Ecology and Systematics 28: 437-466.

Huelsenbeck, J. P., D. M. Hillis, and R. Jones. 1996. Parametric bootstrapping in molecular phylogenetics: applications and performance. Pages 19-45 in J. D. Ferraris and S. R. Palumbi, eds. Molecular zoology: advances, strategies, and protocols. Wiley-Liss, New York.

Kishino, H., and M. Hasegawa. 1989. Evaluation of the maximum likelihood estimate of the evolutionary tree topologies from DNA sequence data, and the branching order of Hominoidea. Journal of Molecular Evolution 29:170-179.

Maldonado-Koerdell, M. 1964. Geohistory and paleogeography of Middle America. Pages 3-32 in R. C. West, ed. Handbook of Middle American Indians. Vol. 1. University of Texas Press, Austin.

McDonald, J. A. 1993. Phytogeography and history of the alpine-subalpine flora of northeastern Mexico. Pages 681-703 in T. P. Ramamorthy, R. Bye, A. Lot, and J. Fa, eds. Biological diversity of Mexico: origins and distribution. Oxford University Press, New York.

Mengel, R. M. 1964. The probable history of species formation in some northern wood warblers (Parulidae). Living Bird 3:9-43.

Moritz, C. 1995. Uses of molecular phylogenies for con- servation. Philosophical Transactions of the Royal Society of London B, Biological Sciences 349:113-118.

Mort, M. E., P. S. Soltis, D. E. Soltis, and M. L. Mabry. 2000. A comparison of three methods for estimating internal support on phylogenetic trees. Systematic Biology 49:160-170.

Page, R. D. M. 1988. Quantitative cladistic biogeography: constructing and comparing area cladograms. Systematic Zoology 37:254-270.

- 1993. COMPONENT, version 2.0. Natural History Museum, London.

- 1994. Maps between trees and cladistic analysis of historical associations among genes, organisms, and areas. Systematic Biology 43:58-77.

Rennert P. D., and C. W. Kilpatrick. 1986. Biochemical systematics of populations of Peromyscus boylii. I. Populations from east-central Mexico with low fundamental numbers. Journal of Mammalogy 67:481-488.

Riddle, B. R. 1995. Molecular biogeography in pocket mice (Perognathus and Chaetodipus) and grasshopper mice (Onychomys): the late Cenozoic development of a North American aridlands rodent guild. Journal of Mammalogy 76:283-301.

. 1996. The molecular phylogeographic bridge between deep and shallow history in continental biotas. Trends in Ecology \& Evolution 11:207-211.

- 1998. The historical assembly of continental biotas: Late Quaternary range-shifting, areas of endemism, and biogeographic structure in the North American mammal fauna. Ecography 21:437-446,

Sullivan, J., and D. L. Swofford. 1997. Are guinea pigs rodents? the importance of adequate models in molecular phylogenetics. Journal of Mammalian Evolution 4: 77-86.

Sullivan, J., K. E. Holsinger, and C. Simon. 1996. The effect of topology on estimates of among-site rate variation. Journal of Molecular Evolution 42:308-312.

Sullivan, J., J. A. Markert, and C. W. Kilpatrick. 1997. Biogeography and molecular systematics of the Peromyscus aztecus group. Systematic Biology 46:426-440.

Swofford D. L., G. J. Olsen, P. J. Waddell, and D. M. Hillis. 1996. Phylogenetic inference. Pages 407-514 in D. M. Hillis, C. Moritz, and B. K. Mable, eds. Molecular systematics. 2d ed. Sinauer, Sunderland, Mass.

Taberlèt, P., L. Fumagalli, A. G. Wust-Saucy, and J. F. Cosson. 1998. Comparative phylogeography and postglacial colonization routes in Europe. Molecular Ecology 7: 453-464.

Templeton, A. 1983. Phylogenetic inference from restriction endonuclease cleavage maps with particular reference to the evolution of humans and the apes. Evolution 37:221-244.

Toledo, V. M. 1982. Pleistocene changes in vegetation in 
tropical Mexico. Pages 93-111 in G. T. Prance, ed. Biological diversification in the Tropics. Columbia University Press, New York.

Van Devender, T. R. 1990a. Late quaternary vegetation and climate of the Chihuahuan Desert, United States and Mexico. Pages 104-133 in J. L. Betancourt, T. R. Van Devender, and P. S. Martin, eds. Packrat middens: the last 40,000 years of biotic change. University of Arizona Press, Tucson.

. 1990b. Late quaternary vegetation and climate of the Sonoran Desert, United States and Mexico. Pages 134-165 in J. L. Betancourt, T. R. Van Devender, and P. S. Martin, eds. Packrat middens: the last 40,000 years of biotic change. University of Arizona Press, Tucson.
Whelan, S., and N. Goldman. 1999. Distribution of statistics used for the comparison of models of sequence evolution in phylogenetics. Molecular Biology and Evolution 16:1292-1299.

Yang Z., N. Goldman, and A. Friday. 1995. Maximum likelihood trees from DNA sequences: a peculiar statistical estimation problem. Systematic Biology 44: 384-399.

Zhang, J. 1999. Performance of likelihood ratio tests of evolutionary hypotheses under inadequate substitution models. Molecular Biology and Evolution 16:868-875.

Zink, R. M. 1996. Comparative phylogeography in North American birds. Evolution 50:308-317.

Associate Editor: Sharon B. Emerson 